\title{
Generation of the rotating light fields under amplitude and phase distortions
}

\author{
Vladimir Volostnikov ${ }^{1}$, Evgeniy Vorontsov ${ }^{1}$, Svetlana Kotova ${ }^{1,2}$, Nikolay Losevsky ${ }^{1}$, \\ Darya Prokopova ${ }^{1,2, *}$, and Sergey Samagin ${ }^{1}$ \\ ${ }^{1}$ Lebedev Physical Institute, Samara, 443011, 221 Novo-Sadovaya Str., Samara, Russia \\ ${ }^{2}$ Samara National Research University, 443086, 34, Moskovskoye shosse, Samara, Russia
}

\begin{abstract}
The work contains study of the influence of amplitude and phase distortions on the generation of two-lobe light fields with the intensity distribution rotating under propagation. Spiral beams, as well as light fields formed on their basis, are considered as particular examples of two-lobe light fields. The amplitude distortions result from the preset different intensity distributions of the illuminating beam, and phase distortions are determined by the primary aberrations.
\end{abstract}

The light fields with a rotating intensity distribution under propagation are the matter of interest in solving various applied problem. One of the bright examples of such problems is the use of two-lobe light fields (i.e. the field with two sharp intensity peaks) [1] for improvement of the longitudinal resolution of fluorescent optical microscopes. The ability of the intensity rotation during propagation is characteristic for spiral light beams [2]. To create these beams you need to set amplitude and phase of the light field that can be implemented by using of the holographic method [3]. However, the latter can not be always used due to the optical system characteristics and low energy efficiency. Therefore a compromise is often made in practice and light fields preserving the spatial structure of their intensity distribution within a limited area, are used instead of the spiral beams. These fields are formed by means of the phase diffractive masks. To calculate the mask phase profile, in our study we used the phase distributions of spiral beams as initial approximation.

Mostly in real optical systems both amplitude and phase distortions occur. For example, in problems of three-dimensional high resolution of the fluorescent microscopy, the intensity distribution of the beam illuminating the phase mask depends on the used micro objective and the ratio of the apertures of the mask and illuminating beam. The phase distortions can be caused by aberrations of the optical system, geometric parameters of the observing system and heterogeneity of the optical parameters of the medium where a light wave propagates. Usually the influence of phase distortions prevails over that of amplitude distortions.

In this paper we continue the research initiated by us earlier in our work [4]. For generation of the light fields, the phase masks based on the spiral beams were used, representing a superposition of Laguerre-Gauss modes. Primary aberrations in the representation of the Zernike polynomials are considered as the phase distortions. Numerical simulation and experiments were performed. A liquid crystal spatial light modulator was used

*Corresponding author: prokopovadv@gmail.com 
as tunable diffractive optical element for light fields formation. Aberrations of different amplitudes have been studied. In figure 1 the phase masks are shown, used for the formation of two-lobes fields with the rotating parameter -2 , for the case of superposition of aberrations with the amplitude of $\lambda / 8$. In figure 2 the results of numerical simulations intensity distribution in focal plane are shown for the case of aberrations of different amplitude. The value of the aberration in the system permissible for the work with this type of fields is estimated. The amplitude distortions were specified by different intensity distributions of the beam illuminating the phase mask.

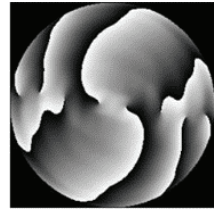

Original mask

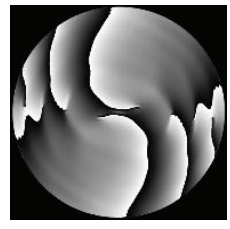

Astigmatism

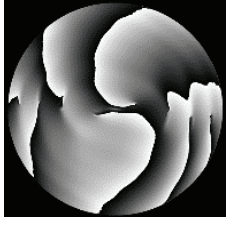

Coma

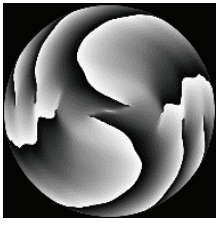

Spherical aberration

Fig. 1. Phase masks for generation of a two-lobe light field with rotating parameter -2 with superposed aberrations of amplitude $\lambda / 8$

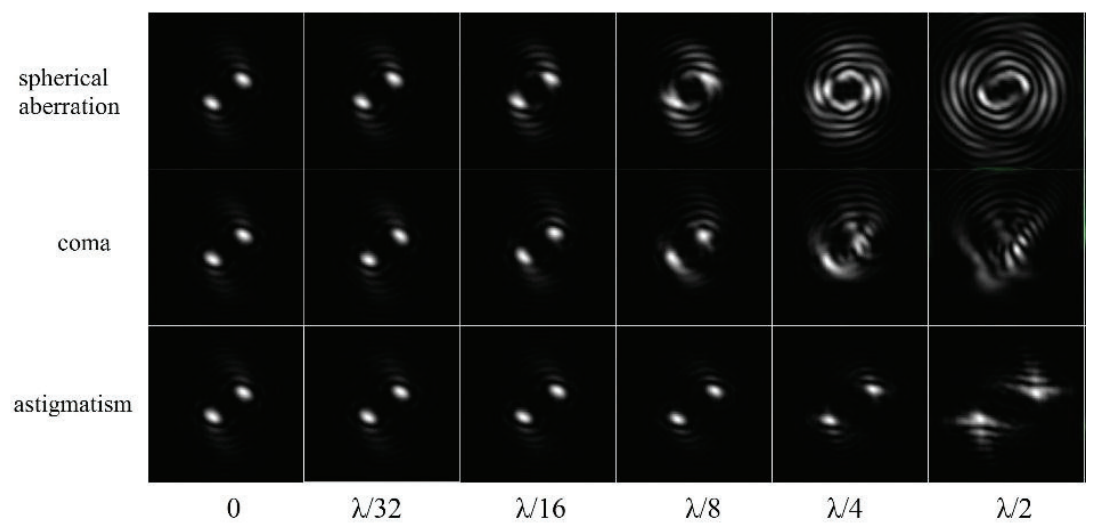

Fig. 2. Calculated intensity distribution in focal plane under the aberration of different amplitude for two-lobe light field with rotating parameter -2 .

The work was supported by the Russian Foundation for Basic Research (Grant No. 16-29-11809) and the Program of Physical Science Department of the Russian Academy of Sciences.

\section{References}

[1] A.V. Naumov, I.Y. Eremchev, A.A. Gorshelev, Eur. Phys. J. D 68, 348 (2014)

[2] E.G. Abramochkin, V.G. Volostnikov, Modern Optics of Gaussian beams (Moscow, Fizmatlit, 2010)

[3] K.N. Afanasiev, S.A. Kishkin, Izvestiya Samarskogo Nauchnogo Tsentra RAN 14, 184 (2012) (in Russian)

[4] E.N. Vorontsov, N.N. Losevsky, D.V. Prokopova, E.V. Razueva, S.A. Samagin, Comp. Opt. 40, 158 (2016) 